\title{
Synthesis and Characterization of Cadmium Sulphide Nanoparticles Using Annona Muricata Leaf Extract as Reducing/Capping Agent
}

\author{
BOKKA DURGA ${ }^{1}$, SHAIK RAZIYA ${ }^{1}$, SANTOSHI G RAJAMAHANTI ${ }^{1}$, \\ BODDETI GOVINDH $^{2}$, KORIMELLA VIJAYA RAJU ${ }^{1}$ and NOWDURI ANNAPURNA ${ }^{{ }^{*}}$ \\ ${ }^{1}$ Department of Engineering Chemistry, AU College of engineering (A), \\ Andhra University, Visakhapatnam, Andhra Pradesh, India \\ ${ }^{2}$ Department of HSS, Raghu Institute of Technology, Visakhapatnam, \\ Andhra Pradesh, India \\ purnaaucevizag@yahoo.co.in
}

Received 23 June 2016 / Accepted 15 July 2016

\begin{abstract}
Cadmium sulphide nanoparticles (CdS) have a great role in chemical research, biotechnology and medicine. The present study mainly involves development of an efficient and environmental friendly method of synthesis of cadmium sulphide nanoparticles by chemical precipitation method using green materials. Annona muricata leaf extract was used as a capping/reducing agent for the preparation of CdS nanoparticles. TEM, XRD, SEM with EDS and UVVis spectroscopy were used to study the morphology, distribution, crystallinity and size of the particle. Biological activity was studied using the bacterial strain staphylococcus aureus and aspergillus niger.
\end{abstract}

Keywords: Annona muricata, Cadmium sulphide nanoparticles, XRD, SEM, TEM and Biological activity

\section{Introduction}

Group II-IV semiconductor nanocrystals such as CdS, CdSe, and CdTe have been studied extensively as they emit in the visible region ${ }^{1,2}$. Many literature reports are available on the application of CdS nanoparticles in the detection of visible radiation, enhancing efficiency of solar cells and a number of biological applications ${ }^{3}$. Due to high stability, excellent physical, chemical and structural properties, availability, ease of preparation and handling, CdS nanomaterials can be exploited in various fields of life. Cadmium sulphide has been widely studied recently ${ }^{4,5}$ and finds many biological applications ranging from molecular histopathology, advanced disease diagnostics to biological imaging ${ }^{6}$.

The synthesis of metal nanoparticles using bio inspired, eco friendly greener methods is one of the most attractive aspects of current nanoscience and nanotechnology Much research work have been reported ${ }^{7,8}$ in the synthesis of nanoparticles using plant extract, bacteria and fungi. It is also found that plants are advantageous for the production of nanoparticles as 
they are easily available, safe to handle and contain a wide range of biomolecules such as alkaloids, terpenoids, phenols, flavonoids, tannins, quinines etc. which mediate the synthesis of nanoparticles 9 .

The leaves of Annona muricata are used for headaches, insomnia, cystitis, liver problems, diabetes, hypertension and as an antiinflammatory, antispasmodic and antidysenteric $^{10,11}$. The decoction of the leaves have parasiticide, antirheumatic and antineuralgic effects when used internally, while the cooked leaves, applied topically, fight rheumatism and abscesses. Very few literature reports are available on the biosynthesis of cadmium sulphide nanoparticles using plant extracts. In view of this and its medicinal importance we have selected Annona muricata leaf extract for the synthesis of cadmium sulphide nanoparticles and an attempt was made on the green synthesis, characterization of structural and morphological features and study of microbial activity.

\section{Experimental}

2 MLH magnetic stirrer REMI, XRD (make PAN analytical, model no: Xpert pro), UVSlimadzu UV-2450 double beam spectrophotometer, FTIR (shimadzu model no: IR prestige-21), SEM (JEOL-JSM model-66IOLV), with EDS (oxford institute), Transmission Electron Microscopy (TEM) were used in this work. Cadmium chloride (AR grade, Qualigens), sodium sulphide (AR grade, Merck), methanol (Merk) were purchased and used.

All the reagents used were highly purified and were used without further purification. The culture media were purchased from Hi- media (India). De-ionized water was employed for preparing all the solutions and reagents.

The dried leaves of Annona muricata plant was washed several times with sterile distilled water to remove all the dirt and dust. The material was dried, cut into small pieces, crushed and powdered. $10 \mathrm{~g}$ of powder was taken in a flask and added $150 \mathrm{~mL}$ water, $10 \mathrm{~mL}$ of methanol then heated to $100{ }^{\circ} \mathrm{C}$ until the volume reduces to half. It is then filtered through Whatman no.1 filter paper and freshly obtained leaf extract was cooled to room temperature and used as such for the synthesis of cadmium sulphide nano particles as a capping/reducing agent.

\section{Preparation of cadmium sulphide nanoparticles}

Synthesis of cadmium sulphide nanoparticles was carried out by using Annona muricata leaf extract by adopting the following procedure.

Annona muricata leaf extract $(5 \mathrm{~mL})$ was added into $50 \mathrm{~mL}$ cadmium chloride $(0.1 \mathrm{M})$ solution. Sodium sulfide $(50 \mathrm{~mL}, 0.1 \mathrm{M})$ dissolved in de-ionized water was added drop wise into the solution of cadmium chloride under magnetic stirring. The contents were later on placed on to a rotatory orbital shaker operating at $200 \mathrm{rpm}, 30{ }^{\circ} \mathrm{C}$ for 12 hours in dark solution. The formation of the particle was monitored by sampling an aliquot $(3 \mathrm{~mL})$ of the mixture after 12 hours, followed by measurement of the UV-Vis spectra using a spectrophotometer. In order to find the absorption maximum, optical density of the content is monitored from wavelength $250-700 \mathrm{~nm}$.

\section{Results and Discussion}

\section{$U V$-Visible spectroscopy}

The UV-Vis spectroscopy gave the absorption spectra of the nanomaterials in the range 200 to $800 \mathrm{~nm}$ of solar spectrum. The absorption edge in bulk CdS is found at $435 \mathrm{~nm}$ while in 
prepared CdS nanoparticles the absorption edges were observed at wavelengths smaller than the bulk which indicated a blue shift in absorption edge. This blue shift was a good agreement with the results reported before ${ }^{12,13}$.

Also shift to lower wavelength (blue shift) in absorption spectra than the bulk was observed in the synthesized CdS nanoparticles as decrease in the particle sizes were observed. The CdS nanoparticles showed blue shift as compared to bulk CdS (435 nm) as shown in the Figure 1.

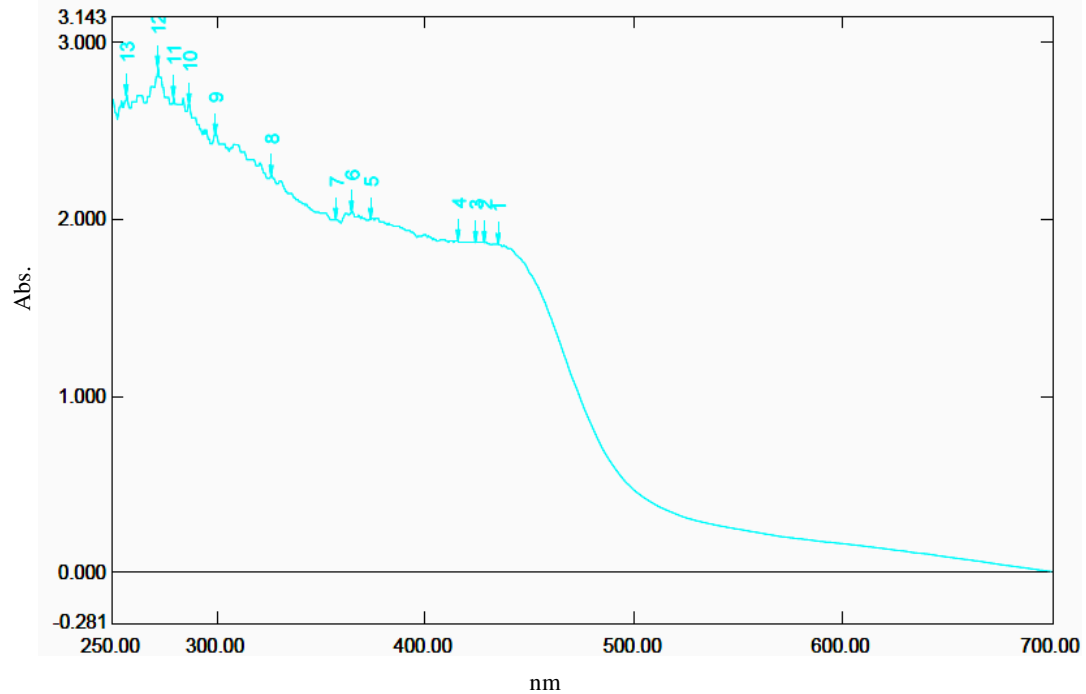

Figure 1. Absorption spectra of $\mathrm{CdS}$ nanoparticles

\section{$X$-Ray diffraction (XRD) spectrometer}

The phase identification and crystalline structures of the nanoparticles were characterized by $\mathrm{x}$-ray powder diffraction. The particle size or grain size of the particles was determined using Scherrer formula. Figure shows the XRD pattern of CdS nanoparticles obtained using Annona muricata leaf extract. The diffraction peaks appeared at 11.61, 14.50, 20.16, 65.16, 67.87 and 79.53 as shown in the Figure 2. The average crystallite size according to Scherrer equation calculated using the highest peak of the 11.61 is found to be $3.42 \mathrm{~nm}$, nearly in agreement with the particle size obtained from TEM Image.

\section{Scanning electron microscopy (SEM)}

SEM measurement was carried out by using JEOL-JSM-66ioLV SEM machine. Thin films of the sample were prepared on a carbon coated copper grid, extra solution was removed using a blotting paper and then the film on the SEM grid were allowed to dry by putting a mercury lamp for $5 \mathrm{~min}$. From the Figure 3 it is clear that the particles are crystalline in nature.

\section{Energy dispersive $x$-ray spectroscopy (EDS)}

Figure 4 reveals the EDS spectra of the synthesized CdS nanoparticles, the presence of Cd and $\mathrm{S}$ peaks confirmed the formation of pure $\mathrm{CdS}$ with no other impurity. Other peaks in this Figure corresponded to carbon, oxygen and chlorine, were due to sputter coating of glass substrate on the EDS stage and were not considered in elemental analysis of $\mathrm{Cd}$ and $\mathrm{S}$. 


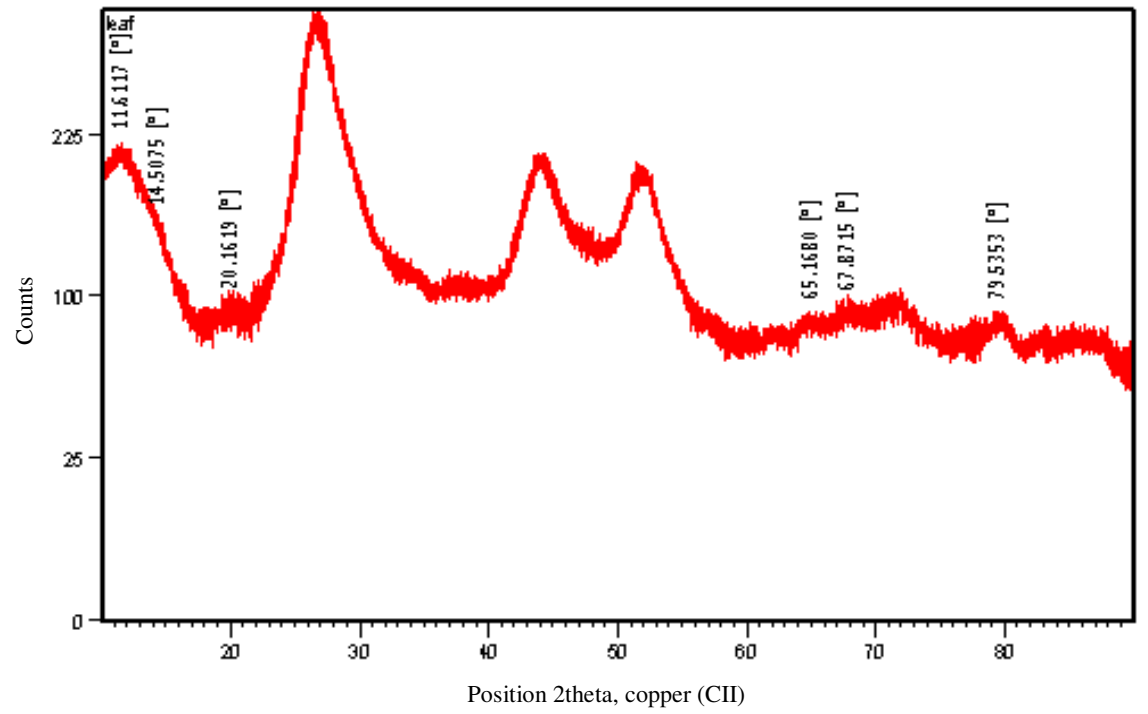

Figure 2. XRD pattern of the CdS nanoparticles

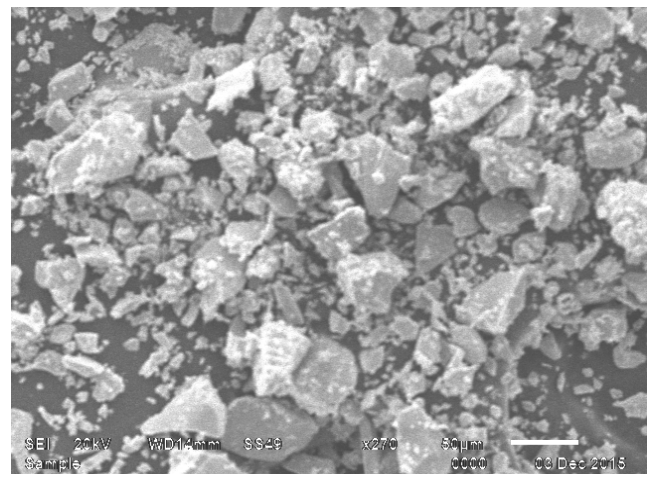

Figure 3. SEM image of CdS nanoparticles

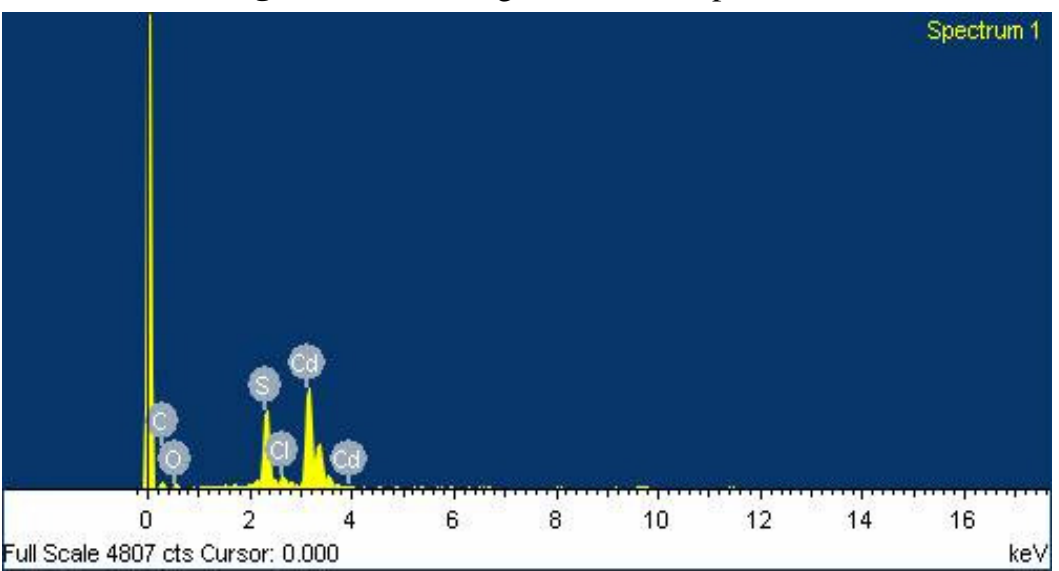

Figure 4. EDS spectra of CdS nanoparticles 


\section{Transmission electron microscopy (TEM)}

TEM analysis is the most reliable method for determining the size of the nano materials. TEM provides the insights in to the morphology, stabilization and the size of the CdS nanoparticles. TEM measurement was carried out to determine the size and morphology of CdS nanoparticles extracellular synthesized from Annona muricata leaf extract. The TEM images obtained for colloid is shown in Figure 5. It is clear from the TEM image (Figure 5) that the particles are spherical in shape and the bar marker represents in the Figure is $4 \mathrm{~nm}$.

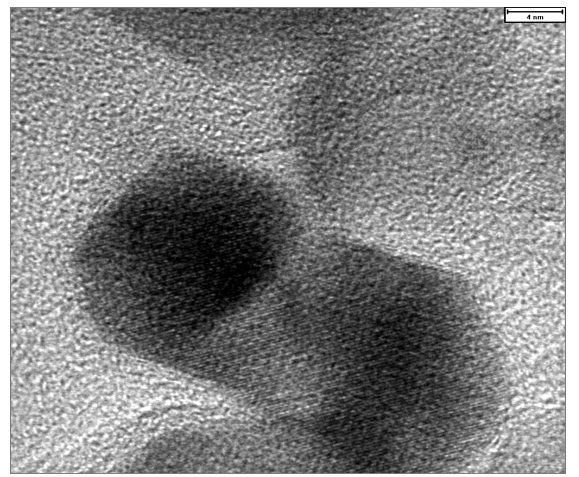

Figure 5. TEM image of CdS nanoparticles

\section{Microbial studies}

\section{Antibacterial activity of the extract and CdS nanoparticle colloid}

The antibacterial activity was examined against bacterial culture (staphylococcus aurues) using standard zone of inhibition assay. Nutrient agar medium was sterilized by moist heat sterilization using an autoclave $\left(121^{\circ} \mathrm{C} ; 15-20 \mathrm{lb}\right.$ for $\left.20 \mathrm{mins}\right)$. 60 Sterile Petri plates were used for the assay to get triplicate values. Molten agar medium was inoculated with microbial suspension and poured in to the plates (temperature of the medium for inoculation is $35-40{ }^{\circ} \mathrm{C}$.) After solidification of the medium, cups were made aseptically using a stainless borer. $50 \mu \mathrm{L}$ of sample of the extracts and an antibiotic solution of $50 \mu \mathrm{L}$ were placed in the cups and the plates were kept in the refrigerator for diffusion for a period of one hour. The plates were then kept in the incubator for one day at $37{ }^{\circ} \mathrm{C}$. The inhibition zone diameters were then recorded and the diameters were compared against those obtained for the standard antibiotic (Figure $6 \& 7$ )

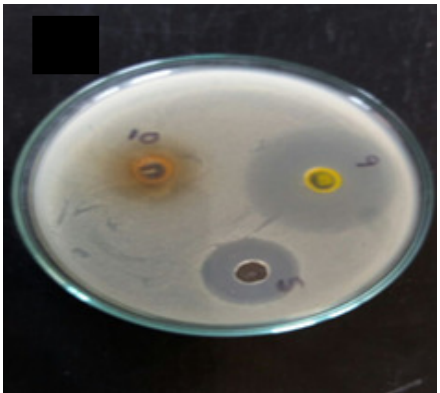

Figure 6. Anti bacterial activity of extract

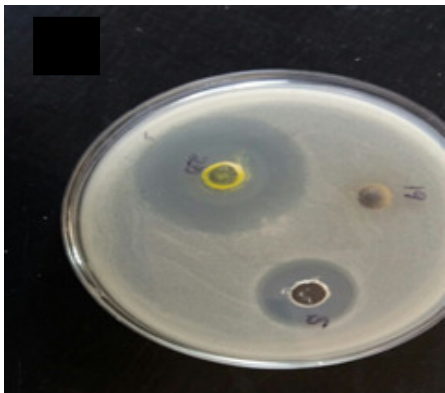

Figure 7. Anti bacterial activity $\mathrm{CdS}$ nanoparticles 


\section{Antifungal activity of the extract and CdS nanoparticle colloid}

The antifungal activity was examined against fungal culture (Aspergillus niger.) using standard zone of inhibition (ZOI) microbiology assay.

Potato dextrose agar medium was sterilized by moist heat sterilization using an autoclave $\left(121{ }^{\circ} \mathrm{C} ; 15-20 \mathrm{lb}\right.$ for $\left.20 \mathrm{mins}\right) .60$ Sterile Petri plates were used for the assay to get triplicate values. Molten agar medium was inoculated with microbial suspension and poured in to the plates (temperature of the medium for inoculation is $35-40{ }^{\circ} \mathrm{C}$.) After solidification of the medium, cups were made aseptically using a stainless borer. $50 \mu \mathrm{L}$ of sample of the extracts and an antibiotic solution of $50 \mu \mathrm{L}$ were placed in the cups and the plates were kept in the refrigerator for diffusion for a period of one hour. The plates were then kept at room temperature for 3 days. The inhibition zone diameters were then recorded and the diameters were compared against those obtained for the standard antibiotic. After 3 days, the inhibition zone diameters were recorded and the diameters were compared with standard antibiotic (Figure 8).

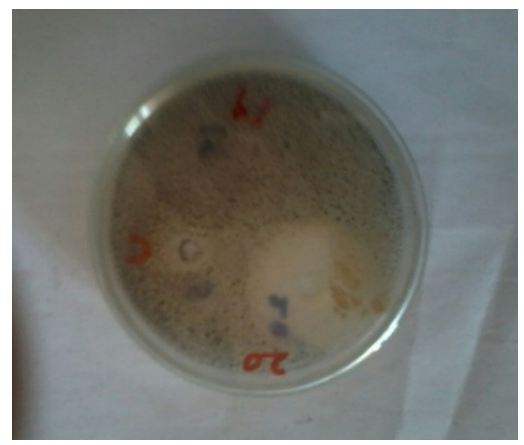

Figure 8. Anti fungal activity of CdS nanoparticles

\section{Conclusion}

In the present study the Annona muricata leaf extract was used for the biosynthesis of CdS nanoparticles and characterized using XRD, SEM and TEM. CdS nanoparticles obtained had properties like spherical shape, as well as particle size distribution. The biological applications such as anti bacterial activity (staphylococcus aurues) and anti fungal activity (Aspergillus niger.) were studied which reveals that the $\mathrm{CdS}$ nanoparticle have excellent anti bacterial and anti fungal activity. But the exact extracellular mechanism of CdS formation with the use of plant extract still remains unclear and requires further detailed investigation.

\section{References}

1. Yu W W, Qu L H, Guo W Z and Peng X G, Chem Mater., 2004, 16(3), 560; DOI:10.1021/cm033007z

2. Aldana J, Wng Y A and Peng X G, J Am Chem Soc., 2001, 123(36), 8844-8850; DOI:10.1021/ja016424q

3. Gupta M and Sharma V, Res J Chem Sci., 2011, 2, 135-138.

4. Alivisatos AP, Science, 1996, 271(5251), 933-937; DOI:10.1126/science.271.5251.933

5. Xue P, Lu R, Huang Y, Jin M, Tac C, Bao C, Wang Z and Zhao Y, Langmuil, 2004, 20(15), 6470-6475; DOI:10.1021/la0493520

6. Jin $\mathrm{S}, \mathrm{Hu} \mathrm{Y}, \mathrm{Gu} \mathrm{Z}$, Lin $\mathrm{L}$ and $\mathrm{Wu} \mathrm{Hu} \mathrm{C}, \mathrm{J}$ Nanomaterial, 2011, 1-13; DOI:10.1155/2011/834139 
7. Bhattacharya D and Gupta R K, Crit Rev Biotechnol., 2005, 25(4), 199-204; DOI:10.1080/07388550500361994

8. Goodsell D S, Bio nanotechnology lessons from nature (Wiley, Hoboken, NT 2004).

9. Kavitha K S, Baker S, Rakshith D, Kavitha H U, Yashwantha Rao H C, Harini B P and Satish S, Int Res J Biological Sci., 2013, 2(6), 66-76.

10. Di Stasi L C, Hiruma-Lima C A, Plantas Medicinais na Amazônia e na Mata Atlântica, $2^{\text {nd }}$ Ed.; Editora UNESP: São Paulo, Brazil, 2002; 87-112.

11. Sousa M P, Matos M E O, Matos F J A, Machados M I L and Craveiro A A, Constituintes Químicos Ativos e Propriedades Biológicas de Plantas Medicinais Brasileiras, $2^{\text {nd }}$ Ed., Editora UFC: Fortaleza, Brazil, 2004; 281-283.

12. Singh V, Sharma P K and Chauhan P, Materials Characterization, 2011, 62(1), 4352; DOI:10.1016/j.matchar.2010.10.009

13. Wang $\mathrm{Y}$ and Herron N, Physical Review B, 1990, 42(11), 7253-7255; DOI:10.1103/PhysRevB.42.7253 